\title{
CIRCLE ACTION AND SOME VANISHING RESULTS ON MANIFOLDS
}

\author{
PING LI AND KEFENG LIU
}

\begin{abstract}
Kawakubo and Uchida showed that, if a closed oriented $4 k$-dimensional manifold $M$ admits a semi-free circle action such that the dimension of the fixed point set is less than $2 k$, then the signature of $M$ vanishes. In this note, by using $G$-signature theorem and the rigidity of the signature operator, we generalize this result to more general circle actions. Combining the same idea with the remarkable Witten-Taubes-Bott rigidity theorem, we explore more vanishing results on spin manifolds admitting such circle actions. Our results are closely related to some earlier results of Conner-Floyd, Landweber-Stong and Hirzebruch-Slodowy.
\end{abstract}

\section{INTRODUCTION AND RESULTS}

Unless otherwise stated, all the manifolds discussed in this paper are closed smooth manifolds and all involutions and circle actions on the manifolds are smooth. We denote by superscripts the corresponding dimensions of the manifolds.

The following is a classical result of Conner and Floyd ([5], §27.2).

Theorem 1.1 (Conner-Floyd). Suppose $g: M^{2 n} \rightarrow M^{2 n}$ is an involution on a manifold and $M^{g}$ is the fixed point set of $g$. If $\operatorname{dim}\left(M^{g}\right)<n$, then the Euler characteristic of $M$ is even.

Here by $\operatorname{dim}\left(M^{g}\right)$ we mean the dimension of highest dimensional connected component of $M^{g}$.

Using their famous $G$-signature theorem, Atiyah and Singer reproved ([3], p.582-p.583) Theorem 1.1 when $n$ is even, $M$ is oriented and $g$ is orientation preserving.

We recall that a circle action ( $S^{1}$-action) is called semi-free if it is free on the complement of the fixed point set or equivalently, the isotropy subgroup of any non fixed point on the manifold is trivial. Using bordism techniques developed by Conner and Floyd in [5], Kawakubo and Uchida showed the following result ([11, Theorem 1.2), which could be taken as a counterpart in the circle case to Theorem 1.1 in some sense.

Theorem 1.2 (Kawakubo-Uchida). Suppose $M^{4 k}$ admits a semi-free $S^{1}$-action and $M^{S^{1}}$ is the fixed point set of this action. If $\operatorname{dim}\left(M^{S^{1}}\right)<2 k$, then the signature of $M, \operatorname{sign}\left(M^{4 k}\right)$, is zero.

Our first purpose in this note is, by closer looking at the $G$-signature theorem in the circle case, to generalize Theorem 1.2 to more general cases. Before stating our first main result, we will introduce some notations, which will be used throughout this paper without further explanation.

2000 Mathematics Subject Classification. 19J35, 57R20, 58J20.

Key words and phrases. circle action, signature, twisted signature operator, rigidity.

The first author is supported by Program for Young Excellent Talents in Tongji University. 
Suppose $M^{2 n}$ is a oriented manifold admitting a $S^{1}$-action. Let $F^{2 m}$ be a connected component of this action. With respect to this $S^{1}$-action, the tangent bundle of $M^{2 n}$ restricted to $F^{2 m},\left.\mathrm{~T} M^{2 n}\right|_{F^{2 m}}$, has the following equivariant decomposition:

$$
\left.\mathrm{T} M^{2 n}\right|_{F^{2 m}}=L_{1} \oplus \cdots \oplus L_{n-m} \oplus \mathrm{T} F^{2 m}
$$

where each $L_{i}$ is a real 2-plane bundle of $F^{2 m}$. We can identify $L_{i}$ with a complex line bundle relative to which the representation of $S^{1}$ on each fiber of $L_{i}$ is given by $e^{\sqrt{-1} \theta} \rightarrow e^{\sqrt{-1} k_{i} \theta}$ with $k_{i} \in \mathbb{Z}-\{0\}$. These $k_{1}, \cdots, k_{n-m}$ are called weights of this $S^{1}$-action on the connected component $F^{2 m}$ and uniquely determined up to signs.

Definition 1.3. Let the notations be as above. We call a $S^{1}$-action prime if there exists a number $\xi \in S^{1}$ such that, for any $k \in \bigcup_{F^{2 m}}\left\{k_{1}, \cdots, k_{n-m}\right\}$, we have $\xi^{k}=-1$.

Remark 1.4. Note that the weights of a semi-free circle action are \pm 1 . Hence semi-free circle actions are prime.

Now we can state our first result, which generalizes Theorem 1.2 ,

Theorem 1.5. Suppose $M^{4 k}$ admits a prime $S^{1}$-action and $M^{S^{1}}$ is the fixed point set of this action. If $\operatorname{dim}\left(M^{S^{1}}\right)<2 k$, then $\operatorname{sign}\left(M^{4 k}\right)=0$.

We will prove this result in Section 2. The signature of a oriented manifold can be realized as an index of some elliptic operator $([3, \S 6)$, now called signature operator. Besides the $G$-signature theorem, the key ingredient of the proof of Theorem 1.5 is the rigidity of the signature operator (see Section 2 for more details). The rigidity of signature operator is only the beginning of a remarkable rigidity theorem: Witten-Taubes-Bott rigidity theorem. Our second purpose in this note is, by using this rigidity theorem, to replace the conclusion of $\operatorname{sign}(M)=0$ in Theorem 1.5 by those of vanishing indices of some twisted signature operators.

In order to state our second result, let us begin with the rigidity of elliptic operators.

Let $D: \Gamma(E) \rightarrow \Gamma(F)$ be an elliptic operator acting on sections of complex vector bundles $E$ and $F$ over a manifold $M$. Ellipticity guarantees that both $\operatorname{ker}(D)$ and $\operatorname{coker}(D)$ are finite-dimensional. Then the index of $D$ is defined as

$$
\operatorname{ind}(D)=\operatorname{dim}_{\mathbb{C}} \operatorname{ker}(D)-\operatorname{dim}_{\mathbb{C}} \operatorname{coker}(D) .
$$

If $M$ admits an $S^{1}$-action preserving $D$, i.e., acting on $E$ and $F$ and commuting with $D$, then both $\operatorname{ker}(D)$ and coker $(D)$ admit an $S^{1}$-action and hence are $S^{1}$-modules. Therefore the virtual complex vector space $\operatorname{ker}(D)-\operatorname{coker}(D)$ has a Fourier decomposition into a finite sum of complex one-dimensional irreducible representations of $S^{1}$ :

$$
\operatorname{ker}(D)-\operatorname{coker}(D)=\sum a_{i} \cdot L^{i},
$$

where $a_{i} \in \mathbb{Z}$ is the representation of $S^{1}$ on $\mathbb{C}$ given by $\lambda \mapsto \lambda^{i}$. The equivariant index of $D$ at $g \in S^{1}$, ind $(g, D)$, is defined to be

$$
\operatorname{ind}(g, D)=\sum a_{i} \cdot g^{i} .
$$

The elliptic operator $D$ is called rigid with respect to this $S^{1}$-action if $a_{i}=0$ for all $i \neq 0$, i.e., $\operatorname{ker}(D)-\operatorname{coker}(D)$ consists of the trivial representation with multiplicity $a_{0}$. Consequently, $\operatorname{ind}(g, D) \equiv \operatorname{ind}(D)$ for any $g \in S^{1}$. An elliptic operator is called universally rigid if it is rigid with respect to any $S^{1}$-action. The fundamental examples of universally rigid operators are 
signature operator and Dirac operator (on spin manifolds). The reason for the former is that both of its kernel and cokernel can be identified with subspaces of the deRham cohomology group $([3], \S 6)$ on which $S^{1}$ always induces a trivial action. The latter is a classical result of Atiyah and Hirzebruch [2].

Let $\Omega_{\mathbb{C}}^{+}$and $\Omega_{\mathbb{C}}^{-}$be the even and odd complex differential forms on a oriented Riemann manifold $M^{2 n}$ under the Hodge *-operator. Then the signature operator

$$
d_{s}: \Omega_{\mathbb{C}}^{+} \rightarrow \Omega_{\mathbb{C}}^{-}
$$

is elliptic and the index of $d_{s}$ equals to $\operatorname{sign}(M)([3], \S 6)$.

Let $W$ be a complex vector bundle over $M$. By means of a connection on $W$, the signature operator can be extended to a twisted operator $([15], I V, \S 9)$

$$
d_{s} \otimes W: \Omega_{\mathbb{C}}^{+}(W) \rightarrow \Omega_{\mathbb{C}}^{-}(W) .
$$

This operator is also elliptic and the index of $d_{s} \otimes W$ is denoted by $\operatorname{sign}(M, W)$.

Let $T_{\mathbb{C}}$ be the complexified tangent bundle of $M$. For an indeterminate $t$, set

$$
\Lambda_{t} T_{\mathbb{C}}=\sum_{k=0}^{\infty} t^{k} \Lambda^{k} T_{\mathbb{C}}, \quad S_{t} T_{\mathbb{C}}=\sum_{k=0}^{\infty} t^{k} S^{k} T_{\mathbb{C}},
$$

where $\Lambda^{k} T_{\mathbb{C}}$ and $S^{k} T_{\mathbb{C}}$ are the $k$-th exterior power and symmetry power of $T_{\mathbb{C}}$ respectively $([1], \S 3.1)$.

Let $R_{i}$ be the sequence of bundles defined by the formal series

$$
\sum_{i=0}^{+\infty} q^{i} R_{i}=\bigotimes_{i=1}^{+\infty} \Lambda_{q^{i}} T_{\mathbb{C}} \otimes \bigotimes_{j=1}^{+\infty} S_{q^{j}} T_{\mathbb{C}}
$$

The first few terms of this sequence are

$$
R_{0}=1, \quad R_{1}=2 T_{\mathbb{C}}, \quad R_{2}=2\left(T_{\mathbb{C}} \otimes T_{\mathbb{C}}+T_{\mathbb{C}}\right),
$$

With all this understood we have the following rigidity theorem.

Theorem 1.6 (Witten-Taubes-Bott). For a spin manifold $M^{2 n}$, each of the elliptic operators $d_{s} \otimes R_{i}$ is universally rigid.

This rigidity theorem was conjectured and given a string-theoretic interpretation by Witten [18]. It was first proved by Taubes [16]. A simper proof was then presented by Bott and Taubes [4]. Using modular invariance of Jacobi functions, the second author gave a more simpler and unified new proof in [13] and further generalized them in [14].

Using this remarkable rigidity theorem, Hirzebruch and Slodowy showed that ([10], p.317), among other things, if $g$ is an involution contained in a circle acting on a spin manifold $M^{4 k}$ and $\operatorname{dim}\left(M^{g}\right)<2 k$, then $\operatorname{sign}\left(M, R_{i}\right)=0$ for all $i$.

We are now ready to state our second main result, which could be taken as the counterpart to Hirzebruch-Slodowy's above mentioned result in the circle case.

Theorem 1.7. Suppose $M^{2 n}$ is a spin manifold admitting a prime $S^{1}$-action. If $\operatorname{dim}\left(M^{S^{1}}\right)<$ $n$, then $\operatorname{sign}\left(M^{2 n}, R_{i}\right)=0$ for all $i$.

Corollary 1.8. Suppose $M^{2 n}$ is a spin manifold admitting a semi-free $S^{1}$-action. If $\operatorname{dim}\left(M^{S^{1}}\right)<$ $n$, then $\operatorname{sign}\left(M^{2 n}, R_{i}\right)=0$ for all $i$. 
Remark 1.9. In [12, Landweber and Stong proved two results concerning the signature and the indices of three twisted Dirac operators, which also have the same feature as our results in some sense. More precisely, they showed that ([12], Theorem 1), if a closed spin manifold $M^{2 n}$ admits a circle action of odd type, then $\operatorname{sign}(M)=0$. Moreover, if this action is semi-free, then $\left([12]\right.$, Theorem 2) $\hat{A}\left(M, T_{\mathbb{C}}\right)=\hat{A}\left(M, \Lambda^{2} T_{\mathbb{C}}\right)=\hat{A}\left(M, \Lambda^{3} T_{\mathbb{C}}+T_{\mathbb{C}}^{2}\right)=0$, where $\hat{A}(M, E)$ is the index of the Dirac operator on $M$ twisted by a complex vector bundle $E$.

\section{Proof of Results}

Let $M^{2 n}$ be a oriented manifold admitting an $S^{1}$-action. Let $F^{2 m}$ be a connected component of the fixed point set $M^{s^{1}}$. As pointed out in Introduction, $\left.\mathrm{T} M^{2 n}\right|_{F^{2 m}}$ can be decomposed into

$$
\left.\mathrm{T} M^{2 n}\right|_{F^{2 m}}=L_{1} \oplus \cdots \oplus L_{n-m} \oplus \mathrm{T} F^{2 m} .
$$

Here $L_{i}$ could be taken as a complex line bundle over $F^{2 m}$ with weight $k_{i}, 1 \leq i \leq n-m$.

$F^{2 m}$ can be oriented so that all orientations of $L_{1}, \cdots, L_{n-m}$ and $F^{2 m}$ taken together yield the orientation of $M^{2 n}$. Let $c_{1}\left(L_{i}\right) \in H^{2}\left(F^{2 m} ; \mathbb{Z}\right)$ be the first Chern class of $L_{i}$. Suppose the total Pontrjagin class of $F^{2 m}$ has the following formal decomposition

$$
p\left(F^{2 m}\right)=1+p_{1}\left(F^{2 m}\right)+\cdots=\prod_{i=1}^{m}\left(1+x_{i}^{2}\right),
$$

i.e., $p_{i}\left(F^{2 m}\right)$ is the $i$-th elementary symmetry polynomial of $x_{1}^{2}, \cdots, x_{m}^{2}$.

With these notations set up, we have the following important lemma, which should be wellknown for experts (cf. [9], §5.8), although, according to the authors acknowledge, nobody state it explicitly as follows.

Lemma 2.1. Let $g$ be an indeterminate. Then the rational function of $g$

$$
\sum_{F^{2 m}}\left\{\left[\left(\prod_{i=1}^{m} x_{i} \frac{1+e^{-x_{i}}}{1-e^{-x_{i}}}\right)\left(\prod_{j=1}^{n-m} \frac{1+g^{k_{j}} e^{-c_{1}\left(L_{j}\right)}}{1-g^{k_{j}} e^{-c_{1}\left(L_{j}\right)}}\right)\right] \cdot\left[F^{2 m}\right]\right\}
$$

identically equals to sign $(M)$. Here $\left[F^{2 m}\right]$ is the fundamental class of $F^{2 m}$ determined by the orientation and the sum is over all the connected components of $M^{S^{1}}$.

Proof. Let $g \in S^{1}$ be a topological generator. Then the fixed point set of the action of $g$ on $M$ are exactly $M^{s^{1}}$. So the $G$-signature theorem ([3], p.582) tells us that

$$
\operatorname{sign}\left(g, M^{2 n}\right)=\sum_{F^{2 m}}\left\{\left[\left(\prod_{i=1}^{m} x_{i} \frac{1+e^{-x_{i}}}{1-e^{-x_{i}}}\right)\left(\prod_{j=1}^{n-m} \frac{1+g^{k_{j}} e^{-c_{1}\left(L_{j}\right)}}{1-g^{k_{j}} e^{-c_{1}\left(L_{j}\right)}}\right)\right] \cdot\left[F^{2 m}\right]\right\}
$$

Here $\operatorname{sign}\left(g, M^{2 n}\right)$ is the equivariant index of the signature operator at $g \in S^{1}$. According to the rigidity of the signature operator, we have $\operatorname{sign}\left(g, M^{2 n}\right) \equiv \operatorname{sign}\left(M^{2 n}\right)$. Therefore (2.1) holds for a dense subset of $S^{1}$ (the topological generators are dense in $S^{1}$ ), which means (2.1) is in fact an identity for an indeterminate $g$.

Remark 2.2. (1) Lemma 2.1 was used in ([9], §5.8), by putting $g=0$, to obtain the famous formula

$$
\operatorname{sign}\left(M^{2 n}\right)=\sum_{F^{2 m}} \operatorname{sign}\left(F^{2 m}\right)
$$


of which several proofs are given by Atiyah-Hirzebruch ([2], §3), Hattori-Taniguchi $([8], \S 4)$, and Witten $([17], \S 3)$ respectively.

(2) When $M^{S^{1}}$ consists of isolated points, Lemma 2.1 was used by Ding (7], p.3947) to obtain some interesting results concerning the representations on the isolated fixed points.

\section{Proof of Theorem 1.5.}

Proof. Now suppose $M^{4 k}$ has a prime $S^{1}$-action. Let $\xi \in S^{1}$ be the desired element as in Definition 1.3. Then we have

$$
\begin{aligned}
{\left.\left[\prod_{j=1}^{2 k-m} \frac{1+g^{k_{j}} e^{-c_{1}\left(L_{j}\right)}}{1-g^{k_{j}} e^{-c_{1}\left(L_{j}\right)}}\right]\right|_{g=\xi} } & =\prod_{j=1}^{2 k-m} \frac{1-e^{-c_{1}\left(L_{j}\right)}}{1+e^{-c_{1}\left(L_{j}\right)}} \\
& =\prod_{j=1}^{2 k-m} \frac{c_{1}\left(L_{j}\right)-\frac{1}{2} c_{1}^{2}\left(L_{j}\right)+\cdots}{2-c_{1}\left(L_{j}\right)+\cdots} \\
& =\left(\prod_{j=1}^{2 k-m} c_{1}\left(L_{j}\right)\right) \cdot \prod_{j=1}^{2 k-m} \frac{1-\frac{1}{2} c_{1}\left(L_{j}\right)+\cdots}{2-c_{1}\left(L_{j}\right)+\cdots} \\
& =e\left(\nu F^{2 m}\right) \cdot \prod_{j=1}^{2 k-m} \frac{1-\frac{1}{2} c_{1}\left(L_{j}\right)+\cdots}{2-c_{1}\left(L_{j}\right)+\cdots},
\end{aligned}
$$

where $e\left(\nu F^{2 m}\right) \in H^{4 k-2 m}\left(F^{2 m} ; \mathbb{Z}\right)$ is the Euler class of the normal bundle of $F^{2 m}$ in $M^{4 k}$.

If $\operatorname{dim}\left(M^{S^{1}}\right)<2 k$, then $4 k-2 m>2 m$, which means $e\left(\nu F^{2 m}\right)=0$ and so by Lemma 2.1 $\operatorname{sign}\left(M^{4 k}\right)=0$. This completes the proof.

\section{Proof of Theorem 1.7.}

Proof. Let $R_{i}$ be the complex vector bundles defined in Introduction. Then for each topological generator $g \in S^{1}$, the equivariant index of the elliptic operator $d_{s} \otimes R_{i}, \operatorname{sign}\left(g, M^{2 n}, R_{i}\right)$, like $G$-signature theorem, could be computed in terms of the local invariants of the fixed point set $M^{S^{1}}$. This is given by a general Lefschetz fixed point formula of Atiyah-Bott-Segal-Singer ([3], p.254-p.258). Instead of writing down the general form of this formula, we only indicate that, for $\operatorname{sign}\left(g, M^{2 n}, R_{i}\right)$, this formula is of the following form.

$$
\sum_{i=0}^{+\infty} q^{i} \cdot \operatorname{sign}\left(g, M^{2 n}, R_{i}\right)=\sum_{F^{2 m}}\left\{\prod_{i=1}^{m}\left[\left(x_{i} \frac{1+e^{-x_{i}}}{1-e^{-x_{i}}}\right) \cdot u_{i}\right] \prod_{j=1}^{n-m}\left[\left(\frac{1+g^{k_{j}} e^{-c_{1}\left(L_{j}\right)}}{1-g^{k_{j}} e^{-c_{1}\left(L_{j}\right)}}\right) \cdot v_{j}\right]\right\} \cdot\left[F^{2 m}\right],
$$

where

$$
u_{i}=\prod_{r=1}^{+\infty} \frac{\left(1+q^{r} e^{-x_{i}}\right)\left(1+q^{r} e^{x_{i}}\right)}{\left(1-q^{r} e^{-x_{i}}\right)\left(1-q^{r} e^{x_{i}}\right)}
$$

and

$$
v_{j}=\prod_{r=1}^{+\infty} \frac{\left(1+q^{r} g^{k_{j}} e^{-c_{1}\left(L_{j}\right)}\right)\left(1+q^{r} g^{-k_{j}} e^{c_{1}\left(L_{j}\right)}\right)}{\left(1-q^{r} g^{k_{j}} e^{-c_{1}\left(L_{j}\right)}\right)\left(1-q^{r} g^{-k_{j}} e^{c_{1}\left(L_{j}\right)}\right)} .
$$

We recommend the readers the references $(([13], \S 1$ and $\S 5)$ or $([6], \S 2.4))$ for a detailed description of $\operatorname{sign}\left(g, M^{2 n}, R_{i}\right)$ in terms of the local data of $M^{S^{1}}$. 
Rigidity theorem 1.6 says that, for an indeterminate $g$, the following identity holds

$$
\sum_{i=0}^{+\infty} q^{i} \cdot \operatorname{sign}\left(M^{2 n}, R_{i}\right) \equiv \sum_{F^{2 m}}\left\{\prod_{i=1}^{m}\left[\left(x_{i} \frac{1+e^{-x_{i}}}{1-e^{-x_{i}}}\right) \cdot u_{i}\right] \prod_{j=1}^{n-m}\left[\left(\frac{1+g^{k_{j}} e^{-c_{1}\left(L_{j}\right)}}{1-g^{k_{j}} e^{-c_{1}\left(L_{j}\right)}}\right) \cdot v_{j}\right]\right\} \cdot\left[F^{2 m}\right],
$$

By using the same idea as in the proof of Theorem 1.5, we can get the conclusion of Theorem 1.7.

\section{Conclusion Remarks}

As we have seen, the key idea in the proofs is to extract a cohomology class $e\left(\nu F^{2 m}\right)$ from the right-hand side of (2.1), by giving a special value on $g$. In fact, for a general compact Lie group $G$ acting on $M^{2 n}$ and $g \in G$, the $G$-signature theorem is of the following form ([3], p.582)

$$
\operatorname{sign}(g, M)=\sum_{F \in M^{g}}\left[e\left(N^{g}(-1)\right) \cdot u\right] \cdot[F],
$$

where $F$ is a connected component of the fixed point set of $g, M^{g}$ (rather than the fixed point set of the whole $G), e\left(N^{g}(-1)\right)$ is the Euler class of a subbundle of the normal bundle on $M^{g}$, corresponding to the eigenvalue -1 of the representation of $g$ on the normal bundle of $F$, and $u \in H^{*}(F)$.

Consequently, the right-hand side of (3.1) vanishes if, for every $F$, the fiber dimension of $N^{g}(-1)$ is greater than the dimension of $F$. This is the corollary 6.13 in [3] on page 582 . It is this corollary that makes Atiyah and Singer to reprove Theorem 1.1 in some cases, because for an involution, -1 is the only eigenvalue on the normal bundle of the fixed point set. While in the circle case, for a topological generator $g=e^{2 \pi \sqrt{-1} \theta} \in S^{1},-1$ in not the eigenvalue $(g$ is a topological generator if and only of $\theta$ is irrational, then $g^{k} \neq-1$ for all weights $k$ ). So we have to construct a cohomology class $\left(e\left(\nu F^{2 m}\right)\right.$ in (2.2) $)$ similar to $e\left(N^{g}(-1)\right)$ in (3.1) artificially. This is the origin of our Definition 1.3 .

\section{REFERENCES}

[1] M.F. Atiyah: K-theory, New-York-Amsterdam, 1967.

[2] M.F. Atiyah, F. Hirzebruch: Spin-Manifolds and Group Actions, 1970 Essays on Topology and Related Topics (Mémoires dédiés à Georges de Rham) pp. 18-28 Springer, New York.

[3] M.F. Atiyah, I.M. Singer: The index theory of elliptic operators: III, Ann. Math. 87 (1968), 546-604.

[4] R. Bott, C. Taubes: On the rigidity theorems of Witten, J. Amer. Math. Soc. 2 (1989), 137-186.

[5] P.E. Conner, E.E. Floyd: Differential periodic maps, Springer, Berlin, 1964.

[6] A. Dessai, R. Jung: On the rigidity theorem for elliptic genera, Trans. Amer. Math. Soc. 350 (1998), 4195-4220.

[7] P. Ding: Topological obstructions to certain Lie group actions on manifolds, Trans. Amer. Math. Soc. 358 (2006), 3937-3967

[8] A. Hattori, H. Taniguchi: Smooth $S^{1}$-action and bordism, J. Math. Soc. Japan. 24 (1972), 701-731.

[9] F. Hirzebruch, T. Berger, R. Jung: Manifolds and modular forms, Aspects of Mathematics, E20, Friedr. Vieweg and Sohn, Braunschweig, 1992.

[10] F. Hirzebruch, P. Slodowy: Elliptic genera, involutions, and homogeneous spin manifolds, Geom. Dedicata. 35 (1990), 309-343

[11] K. Kawakubo, F. Uchida: On the index of a semi-free $S^{1}$-action, J. Math. Soc. Japan. 23 (1971), 351-355.

[12] P. Landweber, R.E. Stong: Circle actions on spin manifolds and characteristic numbers, Topology $\mathbf{2 7}$ (1988), 145-161.

[13] K. Liu: On elliptic genera and theta-functions, Topology. 35 (1996), 617-640.

[14] K. Liu: On modular invariance and rigidity theorems, J. Differential Geom. 41 (1995), 343-396. 
[15] R. Palais: Seminar on the Atiyah-Singer index theorem, Ann. of Math. Stud., vol. 57, Princeton Univ. Press, Princeton, 1965.

[16] C. Taubes: $S^{1}$ actions and elliptic genera, Comm. Math. Phys. 122 (1989), 455-526.

[17] E. Witten: Supersymmetry and Morse theory, J. Differential Geom. 17 (1982), 661-692.

[18] E. Witten: The index of the Dirac operator in loop space, Elliptic curves and modular forms in algebraic topology (Princeton, NJ, 1986), 161-181, Lecture Notes in Math., 1326, Springer, Berlin, 1988.

Department of Mathematics, Tongui University, Shanghai 200092, China

E-mail address: pingli@tongji.edu.cn

Department of Mathematics, University of California at Los Angeles, Los Angeles, CA 90095, USA and Center of Mathematical Science, Zhejiang University, 310027, China

E-mail address: liu@math.ucla.edu 